\title{
Metasurface using multi-layer ceramic capacitors for monostatic radar cross section reduction with broader bandwidth
}

\author{
Thanh Binh Nguyen ${ }^{1}$, Naobumi Michishita ${ }^{1, \text { a) }}$, \\ Hisashi Morishita ${ }^{1}$, Teruki Miyazaki ${ }^{2}$, and Masato Tadokoro ${ }^{2}$ \\ ${ }^{1}$ Graduate School of Science and Engineering, National Defense Academy, \\ Yokosuka-shi, Kanagawa 239-8686, Japan \\ ${ }^{2}$ Yokohama Rubber Co., Ltd.,
}

Hiratsuka-shi, Kanagawa 254-8601, Japan

a)naobumi@nda.ac.jp

\begin{abstract}
In this paper, the metasurface using the multi-layer ceramic capacitors (MLCCs) for monostatic radar cross section (RCS) reduction with broader bandwidth is proposed. By adjusting the width and the thickness of the inner electrodes, and the gap between staggered electrodes of the MLCC, two types of unit cell structures, the reflection phases of which have a phase difference of $180^{\circ}$, are designed. Next, by arranging the MLCCs in checkerboard patterns, the monostatic RCS reductions are simulated. Finally, the simulated results of the reflection phase difference and monostatic RCS reductions are compared with the measured results.
\end{abstract}

Keywords: broader bandwidth, metasurface, multi-layer ceramic capacitor, phase difference, monostatic radar cross section reduction

Classification: Antennas and Propagation

\section{References}

[1] O. Luukkonen, F. Costa, C.R. Simovski, A. Monorchio, and S.A. Tretyakov, "A thin electromagnetic absorbers for wide incidence angles and both polarizations," IEEE Trans. Antennas Propag., vol. 57, no. 10, pp. 3119-3125, Oct. 2009. DOI: 10.1109/tap.2009.2028601

[2] S.A. Tretyakov and S.I. Maslovski, "Thin absorbing structure for all incidence angles based on the use of a high-impedance surface," Microw. Opt. Technol. Lett., vol. 38, no. 3, pp. 175-178, Aug. 2003. DOI: 10.1002/mop.11006

[3] D. Zhirihin, C. Simovski, P. Belov, and S. Glybovski, "Mushroom highimpedance metasurfaces for perfect absorption at two angles of incidence," IEEE Antennas Wireless Propag. Lett., vol. 16, pp. 2626-2629, Aug. 2017. DOI: 10. 1109/lawp.2017.2736506

[4] Y. Zhang, R. Mittra, B.-Z. Wang, and N.-T. Huang, "AMCs for ultra-thin and broadband RAM design," Electron. Lett., vol. 45, no. 10, pp. 484-485, May 2009. DOI: 10.1049/el.2009.3161 
[5] A. Toujo, T. Fujii, C. Kato, Y. Sakabe, and M. Kitano, "Possibility of synthesizing metamaterials with multilayer ceramic capacitors," IEICE General Conf., C-281, March 2008 (in Japanese).

[6] T.-B. Nguyen, N. Kinai, N. Michishita, H. Morishita, T. Miyazaki, and M. Tadokoro, "Dual-polarized metasurface using multi-layer ceramic capacitors for radar cross section reduction," IEICE Trans. Commun., vol. E103-B, no. 8, pp. 852-859, Aug. 2020. DOI: 10.1587/transcom.2019ebp3196

\section{Introduction}

Recently, with the spread of wireless communication technology, various devices such as mobile phones, wearable devices have been diversified and become widespread. Therefore, it is necessary to reduce the influence of antennas of these devices on the human body and suppress unnecessary electromagnetic waves. In recent years, to address these problems, metasurfaces with periodic structures of metal patches [1], mushroom structures [2, 3] have been proposed. However, the bandwidths ( $-10 \mathrm{~dB}$ absorption) of approximately $14.6 \%$ of the metasurface in [1], and approximately $8.7 \%$ of the metasurface in [2] are narrow. Therefore, a combination of patch and loop structures, the reflection phases of which have a phase difference of $180^{\circ}$, were arranged in checkerboard patterns to reduce the radar cross section (RCS) in broadband [4]. However, if the size of the unit cell is large, it will be impossible to arrange them onto a narrow object. Therefore, the size of the unit cell of the metasurface is also required to miniaturize.

In previous studies, the possibility of synthesizing metamaterial using multilayer ceramic capacitor (MLCC) which can make negative permeability has been confirmed [5]. In addition, MLCC is very small, so it can be expected for miniaturizing unit cell of metamaterial. Therefore, a metasurface using MLCCs for RCS reduction has been developed [6]. By arranging MLCCs in checkerboard patterns, the RCS values could be reduced for dual-polarization. In [6], it has been confirmed that only the RCS reductions were obtained at around $29 \mathrm{GHz}$ due to resonance of the MLCC; so the bandwidth of approximately 7.6\% was also narrow. Therefore, the broadband characteristics are required. Furthermore, in [6], when the MLCCs were arranged in the same direction, it has been confirmed that the MLCCs reacted, and the RCS reduction was obtained in transverse electric (TE) incidence. However, in transverse magnetic (TM) incidence, the MLCCs did not react, so it reflected almost like a metal plate. Therefore, when arranging the MLCCs alternately, the reduction characteristics may be obtained by canceling the reflection phases of the adjacent MLCCs. However, it has not been investigated.

In this paper, we propose a metasurface using MLCCs for monostatic RCS reduction with broader bandwidth. By adjusting the width and the thickness of the inner electrodes, and the gap between staggered electrodes of the MLCC, two types of unit cell structures, the reflection phases of which have a phase difference of $180^{\circ}$, are designed. Next, by arranging the MLCCs in checkerboard patterns, the monostatic RCS reductions are simulated. Finally, the simulated results of the reflection phase difference and monostatic RCS reductions are compared with the 
measured results.

\section{MLCC and unit cell structures}

Figure 1(a), (b) show perspective and side views of the MLCC structure. The external dimensions of MLCC and the MLCC structure components are similar to the MLCC in [6]. MLCC consists of dielectric ceramic, plating, barrier, and inner and outer electrodes. The length of the plating and the inner electrodes are 0.28 $\mathrm{mm}$, and $0.585 \mathrm{~mm}$, respectively. The relative permittivity of the internal dielectric ceramic material is 21 . The width, thickness of the inner electrodes are modeled as parameters of $w[\mathrm{~mm}]$ and $t[\mathrm{~mm}]$, and the gap between staggered electrodes is $g$ [mm]. Figure 1(c), (d) show the proposed unit cell structures. Two types of unit cell structure are designed by locating the MLCCs parallel and perpendicular to the incident electric field on an FR-4 substrate. The unit cell size and the FR-4 substrate parameters are also similar to that in [6].

By applying the periodic boundary condition to two types of the unit cell, the

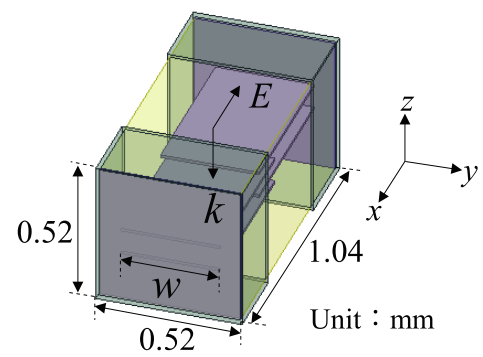

(a)

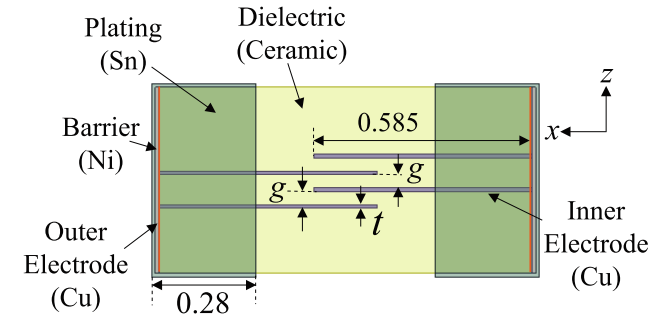

(b)

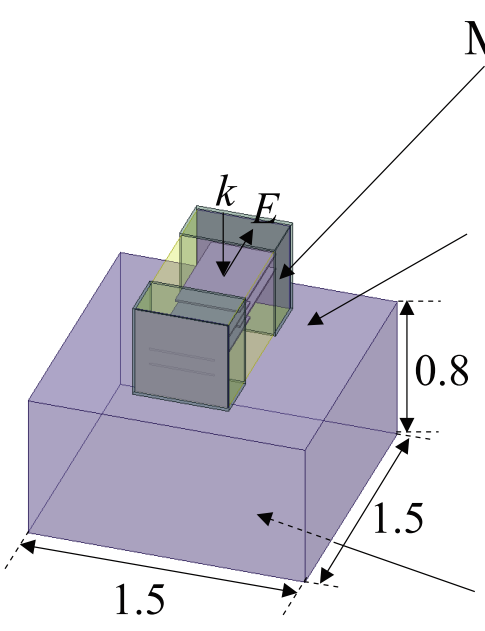

(c)

(d)

Fig. 1. Configurations of MLCC and proposed unit cell structures. (a) Perspective and (b) side views of the MLCC. (c) Unit cell structure in case of the longitudinal direction of the MLCC is parallel to the incident electric field and (d) unit cell structure in case of the longitudinal direction of the MLCC is perpendicular to the incident electric field. 


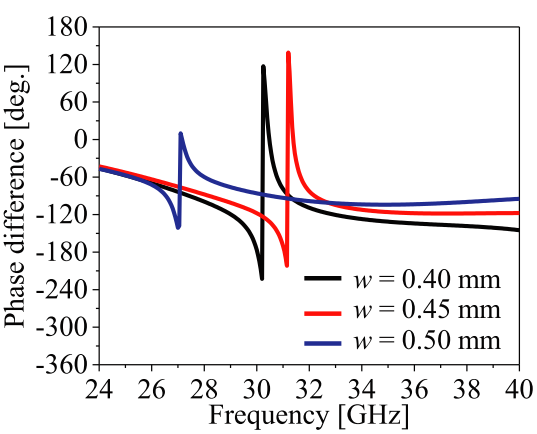

(a)

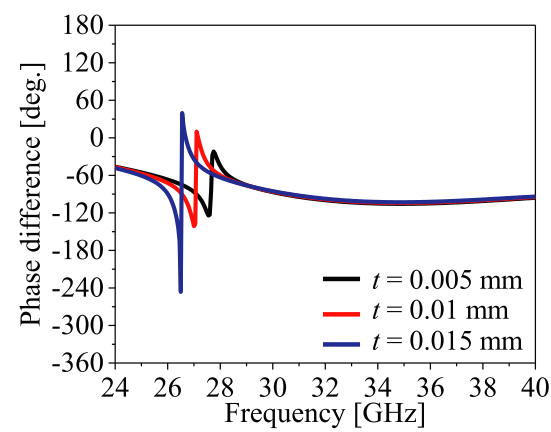

(c)

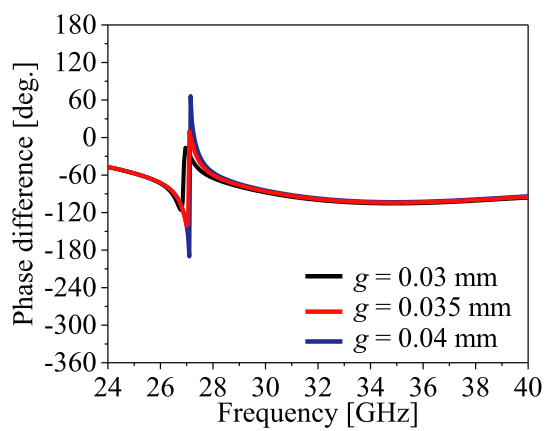

(b)

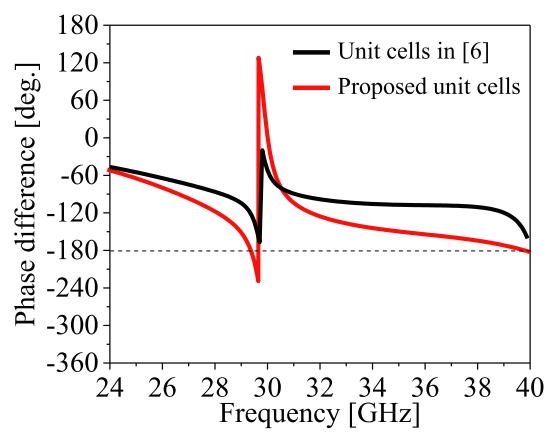

(d)

Fig. 2. Reflection phase difference characteristics when (a) $w$, (b) $g$, and (c) $t$ are varied. (d) Comparison between the optimized reflection phase difference of the proposed unit cell structures and that of unit cells in [6].

reflection phases of them were simulated and the phase difference was calculated. In addition, the backside of the FR-4 substrate was modeled as a perfect electric conductor. Figure 2(a) shows the phase difference characteristics when $g$ of 0.035 $\mathrm{mm}, t$ of $0.01 \mathrm{~mm}$ were fixed, and $w$ was varied from $0.4 \mathrm{~mm}$ to $0.5 \mathrm{~mm}$. The resonant frequency of the MLCC changed, and the phase difference in the high frequency side increased when $w$ decreased. Figure 2(b) shows the phase difference characteristics when $w$ of $0.5 \mathrm{~mm}, t$ of $0.01 \mathrm{~mm}$ were fixed, and $g$ was varied from $0.03 \mathrm{~mm}$ to $0.04 \mathrm{~mm}$. The resonant frequency of the MLCC shifted higher frequency when $g$ increased; however, the phase difference did not change. Figure 2(c) shows the phase difference characteristics when $w$ of $0.5 \mathrm{~mm}, g$ of $0.035 \mathrm{~mm}$ were fixed, and $t$ was varied from $0.005 \mathrm{~mm}$ to $0.015 \mathrm{~mm}$. The resonant frequency of the MLCC shifted lower frequency when $g$ increased; however, the phase difference did not change. Accordingly, the resonant frequency of the MLCC can be controlled by adjusting $t$, and $g$, and the reflection phase difference between two unit cells can be controlled by adjusting $w$. Therefore, to design the unit cell structures in which the MLCC resonates at around $29 \mathrm{GHz}$, and have a phase difference of $180^{\circ}$, the parameters $w, g$, and $t$ of the MLCC were optimized at $0.35 \mathrm{~mm}, 0.035 \mathrm{~mm}$, and $0.01 \mathrm{~mm}$, respectively. Figure 2(d) shows the comparison between the optimized results of the phase difference and that of the unit cells in [6]. The resonant frequency of the proposed MLCC was $29.6 \mathrm{GHz}$, it was the same with that in [6]. However, the reflection phase difference of the proposed unit cell structures was larger than that of 
the unit cells in [6]. It confirmed that the reflection phase difference of approximately $180^{\circ}$ was obtained from $34 \mathrm{GHz}$ to $40 \mathrm{GHz}$.

\section{Comparisons between simulation and measurement results of re- flection phase difference and monostatic RCS reduction character- istics of the proposed metasurfaces}

To validate the simulated results of the reflection phase difference, a finite metasurface structure was fabricated by arranging the MLCCs in the same direction, and the reflection phase of this structure were measured in two cases of TE and TM incidences. In TE incidence, the MLCC resonated at $29.6 \mathrm{GHz}$ and the reflection phase changed suddenly at around the resonant frequency. In addition, in TM incidence, MLCC did not resonate and the reflection phase of this structure was similar to that of the metal plate. Figure 3(a) shows the comparison between the simulated and measured results of the reflection phase difference. The measured reflection phase difference of approximately $180^{\circ}$ was obtained from $34 \mathrm{GHz}$ to $40 \mathrm{GHz}$. It confirmed that the measured results were in good agreement with the simulated results.

By arranging the MLCCs in a checkboard pattern, three types of the finite metasurface prototype divided into $5 \times 5,10 \times 10$, and $20 \times 20$ areas, were fabricated [6]. Figure 3(b), (c) show the simulated and measured results of the monostatic RCS

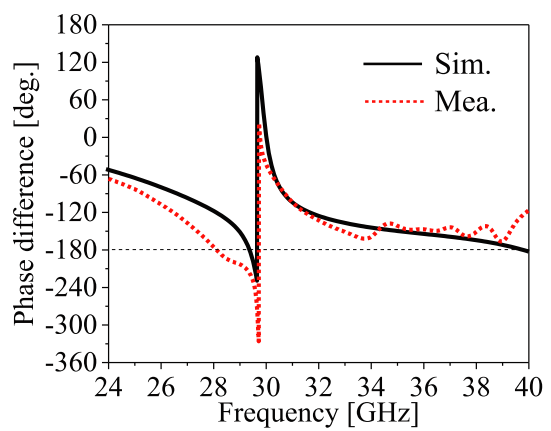

(a)

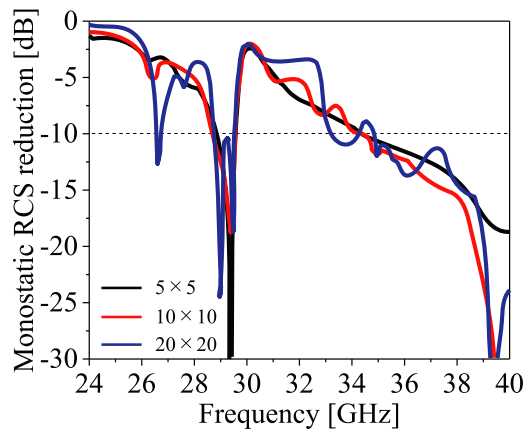

(b)

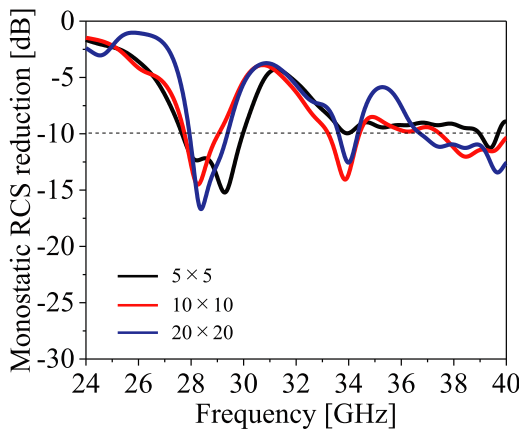

(c)

Fig. 3. (a) Comparison between simulated and measured results of the reflection phase difference. (b) Simulated and (c) measured results of monostatic RCS reduction of the proposed metasurfaces. 
reduction of the proposed metasurfaces. Because the number of MLCCs mounted on the dielectric substrate is large, a surface impedance boundary condition was employed to reduce the computational cost. In addition, the RCS reduction was determined based on the differences among the RCS values for a metal plate with the same dimensions as the metasurface. In simulation results, the monostatic RCS values were reduced at around $29 \mathrm{GHz}$, and the RCS reductions for the $5 \times 5,10 \times$ 10 , and $20 \times 20$ areas of the checkboard patterns were more than $-10 \mathrm{~dB}$ from 34 $\mathrm{GHz}$ to $40 \mathrm{GHz}$. The bandwidth of the proposed metasurface was approximately $16.2 \%$, and it was larger than that in $[1,2,6]$. When a phase difference of $180^{\circ}$ was obtained, the energy was redirected by utilizing the difference in the phase behavior of the two unit cell structures, so the reflected waves were canceled by each other. It was the same with the operating principle of the metasurface in [4]. In addition, the phase difference of approximately $180^{\circ}$ was obtained from $34 \mathrm{GHz}$ to $40 \mathrm{GHz}$, so it could be considered that the RCS reductions were obtained at that frequency band. In measurement results, it confirmed that the monostatic RCS values were also reduced at around $29 \mathrm{GHz}$ and the frequency band from $34 \mathrm{GHz}$ to $40 \mathrm{GHz}$. The RCS reductions from $34 \mathrm{GHz}$ to $40 \mathrm{GHz}$ were smaller than that of simulation results. Since the reacting MLCC has a loss, it could be considered that the level of the reflected wave from the reacting MLCC became small and the amount of the cancellation became small. However, the RCS reductions in measurement results were also approximately $-10 \mathrm{~dB}$, so the measured results were found to approximately agree with the simulated results. Reducing the loss of the reacting MLCCs is future work.

\section{Conclusion}

In this paper, the metasurface using the MLCCs for monostatic RCS reduction with broader bandwidth was proposed. By adjusting the width and the thickness of the inner electrodes, and the gap between staggered electrodes of the MLCC, two types of unit cell structures, the reflection phases of which have a phase difference of $180^{\circ}$, were designed. It confirmed that the reflection phase difference between the proposed unit cell structures was obtained at approximately $180^{\circ}$ from $34 \mathrm{GHz}$ to $40 \mathrm{GHz}$. By arranging the MLCCs in the checkerboard patterns, the monostatic $\mathrm{RCS}$ reductions were simulated and measured. The monostatic RCS reductions were approximately $-10 \mathrm{~dB}$ from $34 \mathrm{GHz}$ to $40 \mathrm{GHz}$ in both the simulated and measured results. The bandwidth of approximately $16.2 \%$ of the proposed metasurface was large, and it confirmed that the measured results were found to approximately agree with the simulated results. 